\title{
Perfil dos pacientes e contexto das consultas em que se fez pela primeira vez o diagnóstico de depressão no Centro de Saúde de Eiras, durante o ano de 2011
}

Patient's profile and the context of consultations in which the diagnose of depression was made for the first time in Eiras Health Centre in 2011

\section{Perfil de losusuarios y contexto de las consultas en que se hizoel primer diagnóstico de depresiónenel Centro de Salud de Eiras durante elaño de 2011}

Paula Miranda. Unidade de Saúde Familiar (USF) VitaSaurium. Soure, Distrito de Coimbra, Portugal. paula.ricardo.miranda@gmail.com (Autora correspondente)

Philippe Botas. UCSP Montemor-o-Velho. Montemor-o-Velho, Distrito de Coimbra, Portugal. phbotas@gmail.com Mariana Abreu. Hospital S. João. Porto, Portugal. marianacgabreu@gmail.com

Carolina Pereira. Unidade de Saúde Familiar (USF) Topázio. Coimbra, Portugal. carolinaduartepereira@gmail.com Luiz Miguel Santiago. Unidade de Saúde Familiar (USF) Topázio, Coimbra; Faculdade de Ciências da Saúde, Universidade da Beira Interior. Portugal. Imsantiago@ netcabo.pt

\section{Resumo}

Objetivo: Este estudo pretendeu conhecer aspectos dos utentes e consultas em que se fez pela primeira vez o diagnóstico de depressão. Métodos: Estudo observacional, transversal e descritivo. População obtida por aplicação de critérios de exclusão aos utentes que, em consulta durante 2011, tiveram em "Avaliação" a codificação "Perturbações depressivas". Pela análise do registro da consulta, estudaram-se as variáveis: idade, sexo, mês, tipo de consulta, consulta presencial/não presencial, sinais/sintomas depressivos anotados e/ou codificados, prescrição de psicofármacos, prescrição pela primeira vez/renovação de receituário. No caso de prescrição pela primeira vez: psicofármacos segundo grupo farmacológico e Denominação Comum Internacional, prescrição de antidepressivo na dose terapêutica e referência ao tempo de tratamento antidepressivo. Resultados: População de 105 indivíduos. Consultas maioritariamente presenciais (79\%). Maior codificação de sinais/sintomas depressivos que anotação apenas ou que anotação e codificação. O sinal/sintoma mais codificado foi "Sensação de depressão" (28\%). Houve prescrição de ansiolíticos isoladamente e um caso de prescrição subterapêutica do antidepressivo. Quanto à duração do tratamento antidepressivo, em 13,7\% das receitas houve menção de que o tratamento deveria prolongar-se no mínimo por 6 meses. Conclusão: A obtenção de uma população pequena e possíveis vieses de informação foram limitações encontradas. Achamos curioso que o sinal/sintoma depressivo mais codificado fosse "Sensação de depressão". É necessário melhorar os registros clínicos e prescrição na depressão.
Palavras-chave:

Depressão

Sinais e Sintomas

Preparações Farmacêuticas Antidepressivos

Ansiolíticos
Como citar: Miranda P, Botas P, Abreu M, Pereira C, Santiago LM. Perfil dos pacientes e contexto das consultas em que se fez pela primeira vez o diagnóstico de depressão no Centro de Saúde de Eiras, durante 0 ano de 2011. Rev Bras Med Fam Comunidade. 2016;11(38):1-9. http://dx.doi.org/10.5712/rbmfc11(38)923
Fonte de financiamento: declaram não haver. Parecer CEP: não se aplica. Conflito de interesses: declaram não haver Procedência e revisão por pares: revisado por pares. Recebido em: 14/04/2014. Aprovado em: 19/04/2016. 


\section{Abstract}

Objective: This investigation intended to know about aspects about patients and consultations in which depression was diagnosed for the first time. Methods: Observational, cross-sectional and descriptive study. Population obtained through the application of exclusion criteria in patients that during 2011 were in "Evaluation" with the codification "Depressive disturbance". Through the analysis of the consultation, we studied the variables: age, sex, month, type of consultation, consultation in the presence of the patient/patient not attending, depressive signs/symptoms noted and/or coded, prescription of psychiatric medication, if it was prescribed for the first time/renewed. In case it is prescribed for the first time: group of medication and name of the drug. If antidepressant was prescribed for the first time: therapeutic dosage/ not and mention the time of treatment. Results: Population of 105 individuals. The consultations were mainly in the presence of the patient (79\%). More encoding of depressive signs/symptoms than annotation only or than annotation and codification. The most coded depressive sign/symptom was "Sensation of depression" (28\%). There was prescription of isolated anxiolytics and a case of under-therapeutic prescription of antidepressant. $13.7 \%$ of the prescription had the reference that the antidepressive treatment should last at least 6 months. Conclusion: Obtainment of a small population and possible information bias were limitations encountered. It is worthy to mention that the most coded depressive sign/symptom was "Sensation of depression". We must improve our clinical records and the prescription in depression.

\section{Resumen}

Objetivo: Este estudio se destinó a comprender los aspectos de los usuarios y las consultas en que se hizo el primer diagnóstico de depresión. Métodos: Se realizó un estudio observacional, transversal y descriptivo. Población obtenida mediante la aplicación de criterios de exclusión a los usuarios que en consulta durante 2011, tuvieran la codificación "trastornos depresivos". Por el análisis de registros de consulta, se estudiaron las variables: edad, sexo, mes, tipo de consulta, consulta presencial/no, signos/síntomas depresivos anotados y/o codificados, la prescripción psicotrópica, si esa prescripción fue por la primera vez/renovación de la prescripción. En el caso de la prescripción por la primera vez: los psicotrópicos según grupo farmacológico y las denominaciones comunes internacionales, referencia, en la prescripción de terapia antidepresiva, a la dosis y tiempo del tratamiento. Resultados: Población de 105 individuos. Sobre todo consultas presenciales (79\%). Más codificación de señales/síntomas depresivos que la anotación sola o que anotación y codificación. El signo/síntoma que se codificó más fue "Sentirse deprimido" (28\%). Fueron prescritos ansiolíticos aislados y hubo un caso de prescripción sub-terapéutica de antidepresivo. En cuanto a duración del tratamiento antidepresivo, en $13,7 \%$ de las prescripciones se mencionaba que el tratamiento debía extenderse por lo menos 6 meses. Conclusión: La obtención de una pequeña población y los posibles sesgos de información fueron limitaciones encontradas. Fue curioso el hecho de que la señal/síntoma más codificada fue "Sentirse deprimido". Es necesario mejorar las historias clínicas y la prescripción en depresión.
Keywords:

Depression

Signs and Symptoms

Pharmaceutical Preparations

AntidepressiveAgents

Anti-AnxietyAgents

Palabras clave:

Depresión

Signos y Síntomas

Preparaciones Farmacéuticas Antidrepresivos

Ansiolíticos

\section{Introdução}

A depressão é uma doença comum, ${ }^{1}$ que afeta maioritariamente o sexo feminino, os grupos etários dos 30 aos 39 anos e dos 50 aos 59 anos, ${ }^{2}$ apresentando-se por vezes com um padrão sazonal. ${ }^{3}$ Estes doentes recorrem principalmente aos Cuidados de Saúde Primários (CSP), ${ }^{2}$ pelo que a depressão é das doenças que um médico de família (MF) mais diagnostica. ${ }^{4}$ Para o diagnóstico, o MF tem que identificar os sinais/sintomas depressivos na entrevista clínica. ${ }^{1}$ Estes são o humor depressivo, anedonia, fadiga, perda de confiança e respeito por si próprio, autocrítica e culpa aumentados, ideias recorrentes de morte ou suicídio ou comportamento suicida, perda de iniciativa, concentração e sentimentos de indecisão, lentificação psicomotora ou excitação, perturbação do sono e alteração do apetite e peso. ${ }^{1}$ É necessária a presença de quatro destes sintomas, num período mínimo de duas semanas, devendo estar presentes pelo menos dois dos três primeiros. É difícil conhecer a frequência de apresentação destes sinais e sintomas, dado que o deprimido tende a não revelar e até esconder as alterações de humor que está vivenciando. ${ }^{1}$

Quanto ao plano, perante um doente deprimido, um MF tem várias abordagens à sua disposição. Uma delas é a farmacológica, tendo-se concluído, num estudo português, que na depressão se opta por prescrever antidepressivos em $49 \%$ dos casos e que a associação de antidepressivo a antipsicótico(s), ocorre em $38 \%$ dos casos. ${ }^{5}$ 
Apesar da posição privilegiada do MF para instituir e monitorizar o tratamento, está descrito que a terapêutica efetuada pelos CSP na depressão é mal conduzida por ser frequente, por exemplo, a prescrição de benzodiazepinas em vez de antidepressivos. ${ }^{2}$ Quando da introdução de antidepressivo, o MF tem de ter em conta que devem iniciar-se com doses dentro dos limites mínimos autorizados e titular, subindo lentamente, dentro dos limites previstos no Resumo de Caraterísticas do Medicamento, ${ }^{6}$ e que o tratamento deve ser mantido durante 6 a 9 meses após a remissão dos sintomas. ${ }^{1}$ Os inibidores seletivos da recaptação da serotonina (ISRS) são frequentemente escolhidos como os fármacos de primeira linha pelo bom perfil antidepressivo aliado à sua segurança e escassez de efeitos secundários. ${ }^{2}$ Em 2007, a Rede de Médicos-Sentinela concluiu que, dentro dos psicofármacos, a fluoxetina era o antidepressivo mais prescrito. ${ }^{5}$ Mais recentemente, em 2009 foi publicada no "The Lancet" uma metanálise que concluía que a sertralina era a melhor opção para iniciar o tratamento da depressão major moderada a severa, dado que comparativamente a outros 11 antidepressivos, apresentava o balanço mais favorável em termos de benefício, aceitabilidade e custo. ${ }^{7} \mathrm{Em} 2009$, ao fim de muitos anos sem haver comercialização de novos antidepressivos, foi lançada uma nova molécula, a agomelatina, um agonista do receptor de melatonina.

Sendo, portanto, a depressão uma condição com a qual o MF lida em grande escala, sobretudo numa altura de crise socioeconômica, interessou-nos estudar a realidade do nosso Centro de Saúde (CS). Pretendemos saber se os nossos deprimidos correspondem, no que diz respeito à idade e sexo, àquilo que literatura descreve e se o diagnóstico é feito sobretudo na primavera e outono. Queremos saber de que sinais/sintomas depressivos se queixam, para aferir se alguns destes apresentam valor preditivo para o diagnóstico. Em relação aos MF do nosso CS, queremos conhecer os seus hábitos de prescrição, segundo grupos farmacológicos, Denominação Comum Internacional (DCI) e as combinações de psicotrópicos que usam. Pretendemos conhecer a sua tendência nos registros clínicos informáticos, se usam mais a redação de texto livre ou antes a codificação ICPC-2 ( $2^{\text {a }}$ edição do ICPC - no original em inglês, International Classification of Primary Care). ${ }^{8}$ Queremos medir a qualidade dos registros clínicos, sendo fator de menor qualidade a ocorrência de diagnósticos pela primeira vez em consultas não presenciais. Desejamos perceber se há falhas na qualidade da prescrição, sendo considerada má prática terapêutica a prescrição subterapêutica de antidepressivo, prescrição de benzodiazepinas isoladamente ou a posologia de antidepressivo por um período de tempo inferior ao definido como necessário para tratar a depressão.

\section{Métodos}

Este é um estudo observacional, transversal e descritivo, cuja coleta de dados decorreu entre maio e julho de 2012. Primeiro, por meio do SAMstat ${ }^{\circledR}$ obteve-se a lista dos utentes que tiveram em consulta registrada eletronicamente no CS, durante 2011, a atribuição do código ICPC-2 "P76- Perturbações depressivas" em Avaliação, "A". A partir daqui, para obtermos a população (utentes do CS com o primeiro diagnóstico de depressão em 2011), consultamos o processo eletrônico $\left(\mathrm{SAM}^{\circledR}\right)$ de cada paciente e excluímos aqueles que tiveram alocação em "A" do código "P76", em consulta decorrida em ano prévio a 2011 e aqueles que tinham em data prévia à consulta registrada em processo eletrônico de 2011 com a primeira Avaliação de "P76": a alocação do código "P03- Sensação de Depressão- P03" ou "P73- Psicose afetiva" em "S" (Subjetivo) ou em "A", qualquer anotação clínica (texto livre redigido pelo MF) com a palavra "depressão" ou palavra derivada ou com os termos "doença maníaco- depressiva", "perturbação 
maníaco-depressiva" ou "doença bipolar" ou qualquer anotação clínica com referência a diagnóstico prévio de depressão.

Em seguida, consultaram-se os processos em papel dos utentes restantes e excluíram-se os utentes transferidos (o seu processo em papel já não está no CS), aqueles cujo processo em papel não se encontrou no arquivo, os utentes em que no processo em papel havia qualquer documento (anotação clínica do MF, carta hospitalar, carta de psiquiatra privado, entre outros) com a palavra "depressão" ou palavra derivada ou os termos "doença maníaco-depressiva", "perturbação maníaco-depressiva" ou "doença bipolar" com data anterior à consulta registrada em processo eletrônico de 2011 com a primeira avaliação de "P76". No caso de na população se encontrar mais do que uma consulta registrada em processo eletrônico em 2011 com a avaliação "P76", a nossa investigação incidiu sobre a primeira a ter ocorrido. Após angariação da população, analisou-se o registro eletrônico da consulta em que foi feito pela primeira vez o diagnóstico de depressão para obtenção dos dados.

Usou-se planilha de cálculo $\left(\right.$ Exce $\left.^{\circledR}\right)$ para registro desses dados e posterior análise estatística. As variáveis estudadas foram a idade, sexo, mês em que decorreu a consulta, tipo de consulta, se consulta presencial/não presencial, sinais/sintomas depressivos anotados e/ou codificados, prescrição de psicofármacos, prescrição pela primeira vez/renovação de receituário. No caso de prescrição pela primeira vez: psicofármacos segundo grupo e $\mathrm{DCl}$, se prescrição de antidepressivo na dose terapêutica e referência ao tempo de tratamento antidepressivo.

Considerou-se sinal/sintoma depressivo codificado qualquer código dos componentes "sinais e sintomas" e "outros diagnósticos" do capítulo "P- Psicológico" e o "sinal e sintoma“ "A04- Debilidade/cansaço geral/fadiga" do capítulo "A- Geral e Inespecífico" que surgisse em "S" da consulta em causa. Os sinais/ sintomas depressivos anotados estavam redigidos em texto livre pelo MF em "S" e/ou em "O" (Objetivo) da consulta em questão. Consideraram-se os termos: depressão, anedonia, anorexia, apatia, cansaço, diminuição de peso, excitação, hipersonia, humor depressivo, indecisão, insónia, isolamento e lentificação. Quando encontramos termos que nos pareceram sinônimos destes, os mesmos foram incluídos, mas especificados em resultados.

O resultado "consulta presencial/não presencial" foi obtido por uma assistente técnica do CS por pesquisa no SINUSSAM ${ }^{\circledR}$. Considerando-se consulta não presencial se lhe foi atribuída no agendamento a cor laranja e presencial se estava agendada a amarelo.

Considerou-se renovação de receituário quando na consulta daquele utente se prescreveram o(s) mesmo(s) psicofármacos já receitados durante o ano anterior à consulta e prescrição pela primeira vez quando pelo menos um psicofármaco não foi prescrito no ano anterior à consulta.

Considerou-se dose terapêutica da agomelatina a ingestão diária entre 25-50mg, de 20-60mg/dia para o citalopram, $60-120 \mathrm{mg} /$ dia para a duloxetina, $10-20 \mathrm{mg} /$ dia para o escitalopram, para a fluoxetina a administração de 20-40mg/dia, ingestão de $20-50 \mathrm{mg} /$ dia de paroxetina e entre $50-200 \mathrm{mg} /$ dia de sertralina, tal como consta nas páginas 31-33 da referência bibliográfica $\mathrm{n} \times 1$. Não havendo nesta, a dose terapêutica da trazodona, a mesma foi definida segundo informação da página 74 da referência bibliográfica $\mathrm{n}^{\circ} 9,{ }^{9}$ sendo de 100-400mg/dia. Não havendo nas anteriores, descrição da dose terapêutica do pirlindol, esta ficou definida como a que é descrita na referência bibliográfica no $10,{ }^{10}$ de 100 a $200 \mathrm{mg} /$ dia.

Esta investigação foi aprovada por Comissão de Ética. 


\section{Resultados}

Obteve-se uma população de 105 indivíduos, composta por 81 mulheres (77,1\%). A população correspondeu a 105 consultas realizadas por $12 \mathrm{MF}$ diferentes. A idade média obtida foi de 47,1 anos, com extremos etários de 88 anos de idade máxima e 9 anos de mínima. 0 mês em que mais se realizou o diagnóstico pela primeira vez de depressão foi setembro, correspondendo a 16 consultas (15,2\%). Em seguida, abril e junho, tendo ocorrido 11 consultas (10,5\%) em cada um destes meses. O diagnóstico pela primeira vez aconteceu em 56 casos no tipo de consulta "Saúde de adultos" (53,3\%). Oitenta e três destas consultas foram presenciais (79\%) vs. $22(21 \%)$ não presenciais.

Não houve anotação e/ou codificação de sinais/sintomas depressivos em 49 consultas (46,7\%), sendo que nas restantes 56 (53,3\%) ocorreu anotação apenas em 11 consultas (19,6\%), em 21 (37,5\%), o MF anotou e codificou os sinais/sintomas depressivos constatados e em 24 consultas (42,9\%) houve apenas codificação. O humor depressivo foi o sinal/sintoma depressivo que surgiu mais vezes anotado (Tabela 1), ao todo 15 vezes, correspondendo a $28,3 \%$.

Tabela 1. Sinais/sintomas depressivos anotados.

\begin{tabular}{|c|c|c|}
\hline & n & $\%$ \\
\hline "Depressão" & 5 & $9,4 \%$ \\
\hline Anedonia & 3 & $5,7 \%$ \\
\hline Apatia (considerados sinônimos: "embotamento", "falta de iniciativa" e "sensação de cabeça oca") & 4 & $7,5 \%$ \\
\hline Cansaço & 2 & $3,8 \%$ \\
\hline Excitação (considerados sinônimos: "stress", "pânico", "ansiedade" e "irritabilidade") & 8 & $15,1 \%$ \\
\hline Hipersonia & 1 & $1,9 \%$ \\
\hline Humor depressivo (considerados sinônimos: “choro”, "emoção”, "tristeza”, "preocupação”, "desesperança” e "angústia”) & 15 & $28,3 \%$ \\
\hline Indecisão & 1 & $1,9 \%$ \\
\hline Insônia & 6 & $11,3 \%$ \\
\hline
\end{tabular}

A "Sensação de depressão", foi codificada 23 vezes, correspondendo a $28 \%$ dos sinais/sintomas depressivos codificados (Tabela 2).

Quanto ao plano, em 80 consultas (76,2\%) houve prescrição de psicofármacos. Em 18 destas (22,5\%) houve renovação de receituário. Nas 62 consultas $(77,5 \%)$ em que houve prescrição de pelo menos um psicofármaco pela primeira vez (Tabela 3), essa prescrição consistiu em 31 casos, em receitar 1 único antidepressivo (50\%), numa consulta $(1,6 \%)$ houve medicalização com associação de antidepressivo e antipsicótico. Dez utentes receberam prescrição unicamente de ansiolítico/sedativo e hipnótico (16,1\%) e duas pessoas levaram receita de antipsicótico apenas $(3,2 \%)$.

As 32 prescrições de psicofármacos pela primeira vez que não antidepressivos consistiram em 29 ansiolíticos/sedativos e hipnóticos (90,6\%) e 3 antipsicóticos (9,4\%). Dentro dos primeiros mencionados, prescreveu-se 26 benzodizepinas, $46,2 \%$ das quais alprazolam $(n=12)$ e 3 extratos de planta- valeriana. 
Tabela 2. Sinais/sintomas depressivos codificados.

\begin{tabular}{lcc}
\hline & $\mathbf{n}$ & $\%$ \\
\hline "A04- Debilidade/cansaço geral/fadiga" & 5 & $6,1 \%$ \\
"P01- Sensação de ansiedade/nervosismo/tensão" & 19 & $23,2 \%$ \\
"P02- Reação aguda/stress" & 5 & $6,1 \%$ \\
"P03- Sensação de depressão" & 23 & $28 \%$ \\
"P06- Perturbação de sono" & 20 & $24,4 \%$ \\
"P29- Sinais/sintomas psicológicos, outros" & 1 & $1,2 \%$ \\
"P74- Distúrbio ansioso/estado ansiedade" & 5 & $6,1 \%$ \\
"P76- Perturbações depressivas" & 4 & $4,9 \%$ \\
Total & 42 & $100 \%$ \\
\hline
\end{tabular}

Tabela 3. Grupos de psicofármacos prescritos pela primeira vez.

\begin{tabular}{lcc}
\hline & N & \% \\
\hline Ansiolítico/sedativo e hipnótico & 10 & $16,1 \%$ \\
Antipsicótico & 2 & $3,2 \%$ \\
Antidepressivo & 31 & 0 \\
Lítio & 16 & $0 \%$ \\
Associação de ansiolítico/sedativo e hipnótico e antidepressivo & 1 & $25,8 \%$ \\
Associação de dois ansiolíticos/sedativos e hipnóticos e um antidepressivo & 1 & $1,8 \%$ \\
Associação de um ansiolítico/sedativo e hipnótico e dois antidepressivos & 1 & $1,8 \%$ \\
Associação de antipsicótico e antidepressivo & 62 & $1,8 \%$ \\
Total & $100 \%$ & \\
\hline
\end{tabular}

Dentro dos antipsicóticos, apenas foram usados neurolépticos de $2^{a}$ geração, mais concretamente, prescreveu-se quetiapina por duas vezes e a risperidona uma vez. Dentro dos antidepressivos prescritos pela primeira vez (Tabela 4), os ISRS foi o grupo mais receitado $(66,7 \%, n=34)$.

Tabela 4. Grupo de antidepressivos prescritos pela primeira vez.

\begin{tabular}{lcc}
\hline & $\mathbf{n}$ & $\%$ \\
\hline Agonista do receptor de melatonina & 2 & $3,9 \%$ \\
Inibidor da monoaminaoxidase tipo A & 7 & $13,7 \%$ \\
Inibidor seletivo da recaptação da serotonina & 34 & $66,7 \%$ \\
Inibidor seletivo da recaptação de serotonina e da noradrenalina & 2 & $3,9 \%$ \\
Tricíclicos e afins & 6 & $11,8 \%$ \\
Total & 51 & $100 \%$ \\
\hline
\end{tabular}

Especificamente por $\mathrm{DCl}$, (Tabela 5) a sertralina foi o antidepressivo mais prescrito pela primeira vez, em 13 casos $(25,5 \%)$.

Dos 51 antidepressivos prescritos pela primeira vez, 31 (60,8\%) foram na sua dose terapêutica, em 19 destas prescrições $(37,7 \%)$ não é possível aferir sobre a adequação posológica dado que o MF não registrou qualquer informação na posologia da receita e há um caso de prescrição subterapêutica (2\%), especificamente de trazodona na dose diária de $50 \mathrm{mg}$. Houve alusão ao tempo de tratamento em sete receitas $(13,7 \%)$ dos antidepressivos prescritos pela primeira vez; em todas elas, o MF mencionou que este se deveria prolongar por pelo menos seis meses. 
Tabela 5. Antidepressivos prescritos pela primeira vez, por DCl.

\begin{tabular}{lcc} 
& N & $\%$ \\
\hline Agomelatina & 2 & $3,9 \%$ \\
Amitriptilina & 1 & $2 \%$ \\
Citalopram & 5 & $9,8 \%$ \\
Duloxetina & 1 & $2 \%$ \\
Escitalopram & 7 & $13,7 \%$ \\
Fluoxetina & 8 & $15,7 \%$ \\
Paroxetina & 1 & $2 \%$ \\
Pirlindol & 7 & $13,7 \%$ \\
Sertralina & 13 & $25,5 \%$ \\
Trazodona & 5 & $9,8 \%$ \\
Venlafaxina & 1 & $2 \%$ \\
Total & 51 & $100 \%$ \\
\hline
\end{tabular}

\section{Discussão}

Uma das limitações deste estudo foi a obtenção de uma população pequena e acreditamos que isto se deveu aos critérios de exclusão da população criados pelos investigadores. Apesar desta pequena população, verifica-se que inúmeros resultados nossos vão ao encontro ao que está descrito na literatura, nomeadamente maior acometimento das mulheres, setembro, mês em que se inicia o outono em Portugal ser aquele em que ocorreu um maior número de diagnósticos, o fato dos ISRS terem sido o grupo de antidepressivos mais prescritos e na prescrição pela primeira vez se ter optado em metade dos utentes pela prescrição unicamente de antidepressivo.

O desenho do nosso estudo proporciona a ocorrência de múltiplos vieses de informação. Nomeadamente, apesar de estar bem definido o grupo de sinais e sintomas necessariamente presentes para fazer o diagnóstico de depressão, cada médico tem uma sensibilidade/intuição diferente para realizar o diagnóstico. Outra fonte de provável viés deve-se a termos consultado registros clínicos eletrônicos de médicos diferentes.

De fato, poucos MF estudaram e poucos dominam a classificação ICPC-2, sendo, por isso, questionável o rigor da utilização destes registros em investigação. ${ }^{11}$ Tentamos reduzir o impacto desta limitação pela inclusão dos sinais/sintomas depressivos redigidos pelo MF em texto livre. Por outro lado, a nossa investigação tem pontos metodológicos relativamente ambíguos como a inclusão de sinais/sintomas depressivos anotados que nos parecessem sinônimos aos termos propostos em metodologia. Para minimizar a variabilidade que daqui pudesse advir, foi sempre o mesmo investigador a levar a cabo este passo da metodologia.

Apesar das suas limitações, achamos que esta investigação é muito pertinente porque põe a nu tópicos da prática clínica dos MF. Concluímos que há aspectos a melhorar. Destacamos alguns resultados a seguir, e refletimos porque terão acontecido. Assim, abrimos portas a novas linhas de investigação, que permitirão perceber realmente porque ocorreram.

Sendo que a depressão se diagnostica por meio da entrevista clínica, ficamos surpresos ao obter este diagnóstico pela primeira vez em consultas não presenciais, em consulta denominada "Receitas" (logo a 
seguir à "Saúde de Adultos") e em consultas em que apenas ocorreu renovação dos psicofármacos que já vinham sendo prescritos no ano anterior. Será que o MF estava a renovar receituário de psicofármacos para outras indicações, por exemplo fibromialgia, profilaxia de enxaqueca ou bulimia, ${ }^{12}$ e pressupôs que por se tratar de psicofármacos o utente sofria de depressão, registando nesse momento erradamente a doença?

Os MF do CS optaram por codificar os sinais/sintomas depressivos mais do que anotar e codificar ou só anotar. Esta tendência já foi descrita noutros estudos. ${ }^{13,14}$ Fica a dúvida se isto significará que os MF do CS sentem domínio/satisfação na utilização da ICPC-2 ou se se deve à subtil imposição da classificação a que foram sujeitos. ${ }^{14}$

A ICPC define que em "S", no motivo de consulta, a identificação do motivo deve ser feita de forma explícita e acordada entre o médico e o doente, que a rubrica da ICPC a utilizar deverá ser tão próxima quanto possível das palavras do doente e que o motivo de consulta é registrado a partir do ponto de vista do doente. ${ }^{13} \mathrm{Um}$ estudo descreveu que na grande parte das consultas o MF não sabe usar a ICPC-2 em "S". ${ }^{13}$ Será que é também o que se passa no nosso CS? É que a codificação mais frequente em motivo de consulta foi "P03- Sensação de depressão", situação muito curiosa sobretudo porque está descrito que os deprimidos tendem a não revelar ou esconder as alterações de humor que vivenciam. ${ }^{1}$

No que concerne à medicação, encontramos situações mencionadas na literatura como de má prática pelo tratamento da depressão com ansiolíticos isoladamente, pelo caso da prescrição de um antidepressivo em dose subterapêutica, sem referência de que este deveria gradualmente ser aumentado na dose. Parece-nos também mais expressiva do que devia a falta de informação por parte do MF na prescrição, dado que em $37,7 \%$ das receitas de antidepressivo, o médico não redigiu nada na posologia.

Quanto ao tempo de tratamento, é um fato que este vinha mencionado em apenas sete receitas de antidepressivo, mas isto, pode-se dever-se à boa prática, muito presente na literatura, de reavaliação clínica do doente a curto prazo, para entre outros, se programar a duração da terapêutica. Em todas as receitas em que o MF colocou o tempo de tratamento antidepressivo, este foi sempre de seis meses no mínimo, o que é insuficiente, dado que um período tão curto não contemplará a fase aguda, de continuação e manutenção do tratamento. ${ }^{6}$

\section{Conclusão}

Obtivemos uma população de 105 deprimidos, sobretudo mulheres e com uma idade média de 47,1 anos. O mês em que mais se fez o diagnóstico foi setembro. Tal como está descrito noutros estudos, os MF do nosso CS optaram mais por codificar os sinais/sintomas depressivos que anotar apenas ou anotar e codificar. O sinal/sintoma depressivo mais codificado foi a "Sensação de depressão" e o humor depressivo o mais anotado. É necessário melhorar a qualidade dos registros clínicos, dado que houve o diagnóstico pela primeira vez de depressão, que deve ser feito por meio de entrevista clínica, em consultas não presenciais. Em 76,2\% das consultas optou-se pela prescrição de psicofármacos. Quando essa prescrição foi pela primeira vez, em metade dos casos consistiu em receitar antidepressivo apenas, principalmente a sertralina. Também no tratamento da depressão há necessidade de melhorar dado que houve prescrição isolada de ansiolíticos/sedativos e hipnóticos, um caso de prescrição em dose subterapêutica de antidepressivo e nas sete receitas com proposta de tempo de tratamento antidepressivo, este foi de seis meses no mínimo, período mais curto do que o mencionado na literatura. 


\section{Agradecimentos}

Os autores gostariam de agradecer aos médicos do CS que permitiram a consulta dos seus registos clínicos e que apoiaram a publicação deste artigo que reflecte sobre a sua actividade clínica e à técnica administrativa Sr. ${ }^{a}$ D. ${ }^{a}$ Catarina Costa que participou na colheita dos dados.

\section{Referências}

1. Isometsä E. Depressão. In: FMUC, Jujitsu e APMCG. Normas de Orientação Clínica. Duodecim Medical Publications; 2009. p. 19-22.

2. Madeira I. Referenciação por depressão à consulta de saúde mental do centro de saúde de Oeiras. Rev Port Clin Geral Fam. 2001;17(2):101-8.

3. Saeed SA, Bruce TJ. Seasonal affective disorders. Am Fam Physician. 1998;57(6):1340-6.

4. Santiago LM, Cobrado NM. Custos directos da terapêutica farmacológica no ambulatório de Clínica Geral. Rev Port Clin Geral Fam. 2002;18(6):351-9.

5. Falcão IM, Monsanto A, Nunes B, Marau J, Falcão JM. Prescrição de psicofármacos em Medicina Geral e Familiar: um estudo na Rede Médicos-Sentinela. Rev Port Clin Geral Fam. 2007;23(1):17-30.

6. Portugal. Ordem dos Médicos. Norma da Direcção Geral da Saúde oㅡ 034/2012, de 30 de dezembro de 2012.

7. Cipriani A, Furukawa TA, Salanti G, Geddes JR, Higgins JP, Churchill R, et al. Comparative efficacy and acceptability of 12 new-generation antidepressants: a multiple-treatments meta-analysis. Lancet. 2009;373(9665):746-58. DOI: http://dx.doi. org/10.1016/S0140-6736(09)60046-5

8. ACSS, APMCG, WONCA. Classificação Internacional de Cuidados de Saúde Primários Segunda Edição. 2a ed. Oxford: Oxford University Press; 2011.

9. Marques-Teixeira J. Consensos psiquiátricos: manual prático para clínicos gerais. Linda-a-Velha: Vale \& Vale Editores; 2007.

10. Portugal. Ministério da Saúde. Serviço Nacional de Saúde. INFARMED.Prontuário terapêutico. Lisboa: Ministério da Saúde; 2011.

11. Braga R. Os registos clínicos e a codificação. Rev Port Med Geral Fam. 2012;28(3):155-6.

12. Martins S. O peso da mente feminina: associação entre obesidade e depressão. Rev Port Clin Geral Fam. 2012;28(3):163-6.

13. Pinto D. Classificar motivos de consulta e procedimentos com a ICPC na prática clínica? Rev Port Med Geral Fam. 2012;28(4):247-8.

14. Melo M. O uso da ICPC nos registos clínicos em Medicina Geral e Familiar. Rev Port Med Geral Fam. 2012;28(4):245-6. 